\title{
QUALIDADE DE VIDA NA TERCEIRA IDADE: UM ESTUDO NAATENÇÃO PRIMÁRIA EM SAÚDE
}

\author{
Melina da Costa Nicolazi ${ }^{1}$, Juliana Krum Cardoso da Silva², Luciana Coelho ${ }^{3}$, Andreia Morales Cascaes ${ }^{4}$, Fátima
}

Büchele $^{5}$

\begin{abstract}
RESUMO: Pesquisa quantitativa com o objetivo de identificar os níveis e a percepção de qualidade de vida entre os idosos em uma comunidade de Florianópolis, em Santa Catarina. Foram entrevistados no domicílio 106 idosos entre 60 e 80 anos de idade, sendo coletadas informações sobre sexo, idade, nível de escolaridade, estado civil e auto-avaliação da saúde e utilizado o instrumento WHOQOL-OLD da Organização Mundial da Saúde para avaliar a qualidade de vida. Dos idosos entrevistados, 73,58\% eram do sexo feminino. A média de idade foi de 69,25 anos. A auto-avaliação negativa da saúde foi referida por 14,15\% dos entrevistados. A qualidade de vida entre a maioria dos idosos investigados apresenta um nível satisfatório. Contudo, em relação ao medo de sentir dor antes de morrer e de a perda dos sentidos afetar de algum modo a vida diária, uma parcela considerável relatou insatisfação, 50\% e 71,7\%, respectivamente.
\end{abstract}

PALAVRAS-CHAVE: Envelhecimento; Idoso; Qualidade de vida.

\section{QUALITY OF LIFE ON ELDERLY: A STUDY ON PRIMARY HEALTHATTENTION}

ABSTRACT: The objective was to identify the levels and perceptions of quality of life among elderly people in a community from the city of Florianópolis in Santa Catarina. A number of 106 older adults between 60 and 80 years were interviewed at home. Information on sex, age, education level, marital status and self-rated health status were collected and the WHOQOLOLD instrument from World Health Organization was used to assess the quality of life. Among the elderly respondents, $73.58 \%$ were female. The average age was of 69.25 years. Poor self-rated health status was reported by $14.15 \%$ of the interviewees. The quality of life among the majority of elderly investigated presents a satisfactory level. However, for the fear pain before death and the impairment to senses affecting daily life, a considerable proportion reported dissatisfaction, $50 \%$ and $71.7 \%$, respectively.

KEYWORDS: Aging; Elderly, Quality of life.

\section{CALIDAD DE VIDA EN LA TERCERA EDAD: UN ESTUDIO EN LAATENCIÓN PRIMARIA EN SALUD}

RESUMEN: Investigación cualitativa con el objetivo de identificar los niveles y la percepción de la calidad de vida entre las personas de edad en una comunidad de Florianópolis, Santa Catarina. Fueron entrevistados en domicilio 106 personas entre 60 y 80 años de edad, siendo recolectadas informaciones sobre sexo, edad, nivel de escolaridad, estado civil y autoevaluación de salud y utilizado el instrumento WHOQOL-OLD de la Organización Mundial de la Salud, para evaluar la calidad de vida. De los ancianos entrevistados, 73,58\% eran de sexo femenino. El promedio de edad fue de 69,25 años. La autoevaluación negativa de la salud fue reportada por 14,15\% de los entrevistados. La calidad de vida entre la mayoría de los ancianos investigados presenta un nivel satisfactorio. Sin embargo, en relación al miedo de sentir dolor antes de morir y la pérdida de los sentidos afectar, de alguna manera, la vida diaria, una proporción considerable relató insatisfacción, $50 \%$ y $71,7 \%$, respectivamente.

PALABRAS CLAVE: Envejecimiento; Anciano; Calidad de vida.

${ }^{1}$ Enfermeira. Atenção Básica do município de Florianópolis-SC.

${ }^{2}$ Enfermeira. Hospital Universitário de Florianópolis-SC.

${ }^{3}$ Enfermeira. TopMed.

${ }^{4}$ Dentista. Atenção Básica do município de Florianópolis-SC. Mestre em Saúde Pública pela Universidade Federal de Santa CatarinaUFSC. Professora Substituta do Departamento de Saúde Pública-UFSC.

${ }^{5}$ Enfermeira. Professora Doutora do Departamento de Saúde Pública-UFSC

Autor correspondente:

Fátima Büchele

Rua Gilmar Darli Vieira, 27 - 88063-650 - Florianópolis-SC

Recebido: 20/05/09

E-mail:buchele@mbox1.ufsc.br

Aprovado: 24/08/09

Cogitare Enferm 2009 Jul/Set; 14(3):428-34 


\section{INTRODUÇÃO}

A Organização Mundial da Saúde-OMS define população idosa como aquela que possui sessenta anos ou mais ${ }^{(1)}$, definição também encontrada no artigo $2^{\circ}$ da Lei 8.842/94 que dispõe sobre a Política Nacional do $\operatorname{Idoso}^{(2)}$.

Segundo dados do Instituto Brasileiro de Geografia e Estatística-IBGE de 2000, o crescimento da população idosa, em números absolutos e relativos, é um fenômeno mundial e está ocorrendo em um nível sem precedência ${ }^{(3)}$. Em 1950, eram cerca de 204 milhões de idosos no mundo e, em 1998, esse contingente alcançou 579 milhões de pessoas, o que indica um crescimento de quase oito milhões por ano. As projeções apontam que, no Brasil, em 2050, a população com 80 anos ou mais será de 13,7 milhões de pessoas, o equivalente à população infantil de zero a 14 anos de idade. O crescimento desta população pode ser atribuído às altas taxas de fecundidade que ocorreram no passado, além da redução da mortalidade entre os idosos ${ }^{(4)}$.

No Brasil, os idosos correspondem a $8,6 \%$ da população total. A região Sul possui 2,3 milhões de idosos, o Estado de Santa Catarina-SC aproximadamente 430 mil e a cidade de FlorianópolisSC um número aproximado de 29 mil idosos, correspondendo a 8,4\% da população total da região ${ }^{(3)}$.

Concomitante ao crescimento da população de idosos surge a necessidade de se investigar fatores relacionados ao processo de envelhecimento. Uma área importante que se tornou objeto de estudo é a qualidade de vida.

A OMS refere que qualidade de vida pode ser entendida como "a percepção que o indivíduo tem de sua posição na vida, dentro do contexto de sua cultura e do sistema de valores de onde vive e em relação a seus objetivos, expectativas, padrões e preocupações"(5:1405). Esta definição reflete a natureza subjetiva dada ao conceito de qualidade de vida que se insere no contexto cultural, social e de meio ambiente. Esse conceito geralmente diz respeito ao bem estar do indivíduo associado a questões como expectativa de vida, paz de espírito, segurança, trabalho, educação, moradia ${ }^{(6)}$.

O Grupo de Qualidade de Vida da Organização Mundial da Saúde-Grupo WHOQOL desenvolveu um instrumento específico para avaliar qualidade de vida em idosos, o World Health Organization Quality of Life Group-WHOQOL-OLD baseado em três aspectos fundamentais: a subjetividade, a multidimensionalidade e a presença de dimensões positivas e negativas ${ }^{(7)}$. Este instrumento apresenta validação transcultural para a língua portuguesa ${ }^{(8-9)}$.

O objetivo do presente estudo foi identificar os níveis e a percepção de qualidade de vida entre os idosos em uma comunidade do município de Florianópolis, em Santa Catarina.

\section{MÉTODOS}

Tratou-se de um estudo transversal, com abordagem quantitativa de caráter exploratório. Foram investigados idosos da área de abrangência de uma Unidade Local de Saúde-ULS do município de Florianópolis, Santa Catarina. A área de abrangência da referida ULS possui cerca de 6.000 habitantes, sendo que destes, 532 (8,76\%) são idosos.

Participaram do estudo 106 idosos entre 60 e 80 anos de idade. As entrevistas foram realizadas no domicílio por três acadêmicas do curso de Graduação em Enfermagem da Universidade Federal de Santa Catarina-UFSC acompanhadas de uma Agente Comunitária de Saúde-ACS, no período de abril a junho de 2008.

Para avaliar a qualidade de vida do idoso, foi utilizado o instrumento World Health Organization Quality of Life Group-WHOQOL-OLD da $\mathrm{OMS}^{(7)}$. O WHOQOL-OLD consiste em 24 itens da escala de Likert atribuídos a seis facetas: Funcionamento do Sensório-FS; Autonomia-AUT; Atividades Passadas, Presentes e Futuras-PPF; Participação Social-PSO; Morte e Morrer-MEM; e, Intimidade-INT. Cada uma das facetas possui 4 itens, portanto, os escores podem variar de 4 a 20.

A faceta Funcionamento do Sensório avalia o funcionamento sensorial e o impacto da perda dessas habilidades na qualidade de vida; Autonomia referese à independência na velhice e, portanto, descreve até que ponto se é capaz de viver de forma autônoma e tomar suas próprias decisões; Atividades Passadas, Presentes e Futuras descreve a satisfação sobre conquistas na vida e coisas a que se anseia; Participação Social delineia a participação em atividades do quotidiano, especialmente na comunidade; Morte e Morrer relacionam-se com preocupações, inquietações e temores sobre a morte e morrer; e Intimidade avalia a capacidade de se ter relações pessoais e íntimas ${ }^{(7)}$.

Para a identificação do entrevistado foi utilizado 
o Questionário de Identificação do Paciente, versão em Português dos Instrumentos de Avaliação de Qualidade de Vida-WHOQOL ${ }^{(10)}$. Com este instrumento, foram coletadas as seguintes variáveis: i) Sexo: masculino e feminino; ii) Idade: coletada de forma contínua e, posteriormente dicotomizada segundo a mediana de distribuição em 60 a 69 anos e 71 a 80 anos de idade; iii) Nível de escolaridade: categorizado em analfabeto, ensino fundamental, ensino médio e ensino superior; iv) Estado civil: casado (a), solteiro (a), separado (a), viúvo (a); v) Autoavaliação da saúde: avaliada através da pergunta: Como está sua saúde?: muito ruim, fraca, nem ruim nem boa, boa e muito boa, posteriormente foi categorizada em positiva, regular e negativa.

O banco de dados foi construído e analisado no programa estatístico Stata 9. Realizou-se estatística descritiva por meio da distribuição de frequência e de medidas de tendência central e dispersão das variáveis estudadas.

O projeto de pesquisa foi aprovado pelo Comitê de Ética em Pesquisa com Seres Humanos da Universidade Federal de Santa Catarina, sob o número 011/08 com obtenção do Termo de Consentimento Livre e Esclarecido de todos os entrevistados.

\section{RESULTADOS}

Participaram do estudo 106 idosos entre 60 e 80 anos, correspondendo a $20 \%$ do total de idosos da área de abrangência da ULS estudada.

\section{Características pessoais dos entrevistados}

Dos idosos entrevistados 73,58\% ( $n=78$ ) eram do sexo feminino e $26,42 \%$ ( $n=28$ ) do sexo masculino. A média da idade foi de 69,25 anos e a mediana 69 anos. A maioria dos idosos estudou até o ensino fundamental (73,58\%), enquanto $4,72 \%$ não foram alfabetizados. Metade dos entrevistados é casada, enquanto mais de um terço da amostra encontra-se viúvo (38,68\%). A maior parte dos idosos auto-avalia sua saúde como regular $(45,28 \%)$ e $14,15 \%$ apresentaram auto-avaliação negativa do estado de saúde (Tabela 1).

Tabela 1 - Frequência e porcentagem de distribuição das características sócio-demográficas dos idosos entrevistados ( $n=106)$. Florianópolis-SC, 2008

\begin{tabular}{|c|c|c|}
\hline Características & $\mathrm{N}$. & $\%$ \\
\hline \multicolumn{3}{|l|}{ Sexo } \\
\hline Masculino & 28 & 26,42 \\
\hline Feminino & 78 & 73,58 \\
\hline \multicolumn{3}{|l|}{ Idade } \\
\hline $60-69$ & 58 & 54,72 \\
\hline $71-80$ & 48 & 45,28 \\
\hline \multicolumn{3}{|l|}{ Nível de escolaridade } \\
\hline Analfabeto & 5 & 4,72 \\
\hline Ensino Fundamental & 78 & 73,58 \\
\hline Ensino Médio & 17 & 16,04 \\
\hline Ensino Superior & 6 & 5,66 \\
\hline \multicolumn{3}{|l|}{ Estado Civil } \\
\hline Solteiro(a) & 3 & 2,83 \\
\hline Casado(a) & 53 & 50,00 \\
\hline Separado(a) & 9 & 8,49 \\
\hline Viúvo(a) & 41 & 38,68 \\
\hline \multicolumn{3}{|l|}{ Auto-avaliação da saúde } \\
\hline Boa & 43 & 40,57 \\
\hline Regular & 48 & 45,28 \\
\hline Ruim & 15 & 14,15 \\
\hline
\end{tabular}

\section{Qualidade de vida dos idosos entrevistados}

A Tabela 2 apresenta a distribuição das respostas de cada faceta do questionário WHOQOLOLD. Em relação à faceta Funcionamento do Sensório-FS, a maior parte da população pesquisada relata não apresentar perdas sensoriais que possam afetar sua participação em atividades $(71,7 \%)$ e interação com outras pessoas (79,2\%). Entretanto, quase a metade relatou que as perdas sensoriais podem afetar sua vida diária de algum modo, enquanto que a avaliação negativa dos sentidos foi reportada por quase $15 \%$ dos entrevistados. Na faceta Autonomia-AUT observa-se que cerca de um quarto dos idosos pesquisados não consegue realizar as coisas que gostaria e nem controlar seu futuro, contudo, mais de $80 \%$ demonstra que possui bastante ou extrema liberdade e que as pessoas respeitam essa liberdade.

Os dados relativos às atividades Passadas, Presentes e Futuras-PPF demonstram que 11,3\% dos pesquisados sentem que tiveram muito pouco reconhecimento merecido na vida e 13,2\% encontramse muito pouco satisfeitos com oportunidades para outras realizações, enquanto que cerca de $70 \%$ está 
feliz ou muito feliz com as coisas a esperar daqui para frente e a maioria encontra-se satisfeita $(56,6 \%)$ muito satisfeita (25,5\%) com as realizações na vida.

Ao avaliar a faceta Participação Social-PSO, observou-se que cerca de $70 \%$ indicaram satisfação ou muita satisfação com a maneira de usar seu tempo e com o seu nível de atividade, e cerca da metade dos idosos sentem que têm o suficiente para fazer no seu cotidiano e afirmam-se satisfeitos em participar das atividades na comunidade. A percepção dos idosos quanto a Morte e Morrer-MEM revelou que a maioria $(74,5 \%)$ não tem medo de morrer e nem se preocupa com a maneira pela qual irá morrer, porém, cerca de
50\% têm medo de sofrer de dor antes de morrer. As questões relacionadas à Intimidade-INT demonstraram que 67\% dos indivíduos da amostra têm oportunidades de amar e ser amado, entretanto quase $15 \%$ afirmaram possuir nenhum ou muito pouco companheirismo na vida.

Observou-se ainda que as facetas Funcionamento do Sensório-FS e Morte e MorrerMEM apresentaram médias semelhantes, enquanto que Intimidade-INT e Atividades Presentes, Passadas e Futuras-PPF apresentaram médias ligeiramente maiores do que Autonomia-AUT e Participação Social-PSO.

Tabela 2 - Distribuição das respostas, média, mediana e desvio padrão dos itens do instrumento WHOQOL-OLD aplicado em idosos ( $n=106)$. Florianópolis-SC, 2008

\begin{tabular}{|c|c|c|c|c|c|c|c|c|c|}
\hline & \multirow[t]{2}{*}{ Facetas } & \multicolumn{5}{|c|}{ Respostas (\%) } & \multirow[t]{2}{*}{ Média } & \multirow[t]{2}{*}{ Mediana } & \multirow[t]{2}{*}{$\mathrm{DP}$} \\
\hline & & 1 & 2 & 3 & 4 & 5 & & & \\
\hline \multirow[t]{4}{*}{ FS } & Afetar a vida diária & 52,8 & 17,0 & 16,0 & 14,2 & 0,0 & 1,92 & 1,00 & 1,12 \\
\hline & Afetar atividades diárias & 71,7 & 7,6 & 7,6 & 13,2 & 0,0 & 1,62 & 1,00 & 1,09 \\
\hline & Afetar interação com pessoas & 79,2 & 5,7 & 9,4 & 5,7 & 0,0 & 1,42 & 1,00 & 0,88 \\
\hline & Funcionamento dos sentidos & 6,6 & 7,6 & 34,0 & 46,2 & 5,7 & 2,63 & 2,00 & 0,95 \\
\hline \multirow[t]{6}{*}{ AUT } & Liberdade para tomar decisões & 0,9 & 4,7 & 13,2 & 55,7 & 25,5 & 4,00 & 4,00 & 0,82 \\
\hline & Controle do futuro & 8,5 & 17,9 & 25,5 & 46,2 & 1,9 & 3,15 & 3,00 & 1,02 \\
\hline & Pessoas respeitam liberdade & 1,9 & 2,8 & 9,4 & 70,8 & 15,1 & 3,94 & 4,00 & 0,73 \\
\hline & Faz o que gostaria & 2,8 & 21,7 & 25,5 & 36,8 & 13,2 & 3,36 & 3,50 & 1,05 \\
\hline & Satisfação com oportunidades & 2,83 & 11,3 & 23,6 & 52,8 & 9,4 & 3,55 & 4,00 & 0,92 \\
\hline & Reconhecimento merecido & 3,8 & 13,2 & 19,8 & 55,7 & 7,6 & 3,50 & 4,00 & 0,95 \\
\hline \multirow[t]{4}{*}{ PPF } & Satisfação com o que alcançou & 0,9 & 5,7 & 11,3 & 56,6 & 25,5 & 4,00 & 4,00 & 0,83 \\
\hline & Felicidade com o que esperar & 0,0 & 3,8 & 25,5 & 64,2 & 6,6 & 3,74 & 4,00 & 0,64 \\
\hline & Tem o suficiente para fazer & 0,0 & 9,4 & 32,1 & 50,9 & 7,6 & 3,57 & 4,00 & 0,77 \\
\hline & Satisfação com a maneira de usar o tempo & 1,9 & 10,4 & 12,3 & 57,6 & 17,9 & 3,79 & 4,00 & 0,92 \\
\hline \multirow[t]{3}{*}{ PSO } & Satisfação com o nível de atividade & 1,9 & 11,3 & 17,0 & 55,7 & 14,2 & 3,69 & 4,00 & 0,92 \\
\hline & $\begin{array}{l}\text { Oportunidades com atividades na } \\
\text { comunidade }\end{array}$ & 2,8 & 8,5 & 28,3 & 52,8 & 7,6 & 3,54 & 4,00 & 0,86 \\
\hline & Preocupação com a maneira de morrer & 74,5 & 7,6 & 10,4 & 6,6 & 0,9 & 1,51 & 1,00 & 0,98 \\
\hline \multirow[t]{5}{*}{ MEM } & Medo de não controlar sua morte & 70,8 & 8,5 & 10,4 & 8,5 & 1,9 & 1,62 & 1,00 & 1,09 \\
\hline & Medo de morrer & 74,5 & 7,6 & 10,4 & 6,6 & 0,9 & 1,52 & 1,00 & 0,99 \\
\hline & Quanto teme sofrer de dor & 26,4 & 8,5 & 12,3 & 49,1 & 3,8 & 3,04 & 2,00 & 1,34 \\
\hline & Companheirismo na vida & 1,9 & 12,3 & 16,0 & 61,3 & 8,5 & 3,62 & 4,00 & 0,88 \\
\hline & Amor na vida & 1,9 & 2,8 & 9,4 & 71,1 & 14,2 & 3,93 & 4,00 & 0,72 \\
\hline \multirow[t]{2}{*}{ INT } & Oportunidades de amar & 3,8 & 0,9 & 14,2 & 67,0 & 14,2 & 3,87 & 4,00 & 0,81 \\
\hline & Oportunidades para ser amado & 0,9 & 1,9 & 18,9 & 67,9 & 10,4 & 3,83 & 4,00 & 0,71 \\
\hline
\end{tabular}

Funcionamento do Sensório-FS, Autonomia-AUT, Atividades Passadas, Presentes e Futuras-PPF, Participação Social-PSO, Morte e Morrer-MEM e Intimidade-INT 


\section{DISCUSSÃO}

O processo do envelhecimento humano vem seguido de modificações biológicas e psicológicas, ou seja, de um desgaste físico e funcional do corpo e da mente, assim como de uma redução das respostas fisiológicas às ações do ambiente em que o idoso está inserido. Portanto, avaliar a qualidade de vida na velhice implica na adoção de múltiplos critérios de natureza biológica, psicológica e sócio-estrutural ${ }^{(11)}$.

O instrumento de avaliação da qualidade de vida proposto pelo Grupo de Qualidade de Vida da Organização Mundial da Saúde-WHOQOL-OLD apresenta os principais elementos apontados como determinantes ou indicadores de bem-estar na velhice: bom funcionamento do sensório, autonomia, satisfação sobre conquistas na vida e coisas a que se anseia, participação em atividades, não se preocupar com a morte e o morrer e possuir boas relações pessoais e íntimas ${ }^{(11)}$.

No presente estudo, quase a metade dos idosos entrevistados relatou que as perdas sensoriais afetavam a sua vida diária. A diminuição dos sentidos é uma característica apresentada em grande parcela dos idosos nas populações. As papilas gustativas geralmente diminuem em quantidade ou atrofiam e a produção de saliva também reduz, afetando o paladar. Já a visão, pode ser acometida por doenças como a vista cansada, alterações nas pupilas, cataratas e o glaucoma, podendo ocasionar um comprometimento da autonomia do idoso ${ }^{(12)}$. O sentido da audição por sua vez pode comprometer a comunicação, a realização de atividades, a interação social ${ }^{(13)}$.

Entretanto, é importante que o processo de envelhecimento seja enfrentado de forma construtiva e participativa, com reforço da autonomia e não apenas somando perdas nas dimensões de capacidades físicas ou intelectuais, mas dinamizando ao máximo os aspectos positivos $^{(14)}$.

Com relação à autonomia, sua perda representa, para muitos idosos, uma das maiores preocupações além de perda na qualidade de vida. Para esta população a saúde está relacionada diretamente com independência, capacidade para fazer as coisas, trabalhar, poder ir e vir, mesmo portando algumas doenças crônicas. Mantendo-se independentes e autônomos, as dificuldades serão menores, tanto para eles, quanto para as famílias e a sociedade ${ }^{(15)}$. Boa parte dos idosos da presente pesquisa não se sente plenamente satisfeita com relação à possibilidade de fazer as coisas que gostariam. Isto pode ocorrer devido à impossibilidade física, financeira ou por terem deixado o mercado de trabalho ${ }^{(16)}$. Neste último caso é importante que o idoso seja estimulado a procurar atividades que lhe agradem e ocupem seu tempo, para não se sentir incapaz, improdutivo.

Para os idosos é muito importante ter independência e autonomia em suas ações, tomar suas próprias decisões e, principalmente, estas serem respeitadas pelas pessoas ao redor. Sentir que possui o controle de sua vida gera ao idoso a possibilidade de fazer projeções para o futuro, o que o torna mais esperançoso e feliz melhorando, assim, a sua qualidade de $\operatorname{vida}^{(17)}$. Em relação às atividades passadas, presentes e futuras, uma grande parte dos idosos do presente estudo sente-se satisfeita com o que conquistaram ao longo da vida e com o reconhecimento recebido por isto.

Uma qualidade de vida satisfatória na velhice não é um atributo apenas do indivíduo biológico, psicológico ou social, mas resulta também da qualidade da interação entre pessoas em mudança, vivendo em uma sociedade que está em constante mudança ${ }^{(11)}$. A interação social é muito importante na velhice, pois esta é uma fase da vida na qual normalmente os indivíduos acabam se isolando e se excluindo do contexto social ${ }^{(18)}$. Os resultados do presente estudo demonstram que uma parcela considerável da população investigada não está plenamente satisfeita com as atividades que realiza e com as atividades que participa na comunidade em que está inserida. A exclusão social pode ser atribuída a uma questão cultural em que algumas sociedades consideram os idosos antiquados, pouco hábeis, diminuindo seu valor, tornando-os incapazes de desfrutar dos prazeres da vida ${ }^{(19)}$.

Algumas oportunidades de contato e interação social podem ser encontradas em ações coletivas para a Terceira Idade em parceria com outros atores sociais como, por exemplo, participação em grupos no centro comunitário, grupos de atividade física ${ }^{(18)}$. Esta última está associada com a melhora da saúde e com a redução da morbidade e da mortalidade, além de proporcionar melhoras nos aspectos psicológicos e sociais das pessoas que a praticam regularmente ${ }^{(20)}$.

A velhice é caracterizada por uma redução nos contatos sociais, que reflete uma seleção ativa na qual as relações sociais emocionalmente próximas são mantidas porque são mais importantes para a adaptação do idoso. Um estudo ${ }^{(21)}$ observou que o número de parceiros sociais até pode ser estável ao longo da vida, 
mas o número de relações sociais periféricas declina na velhice. Os idosos que reduzem os contatos periféricos, mas mantém contatos emocionais significativos com pessoas afetivamente próximas, desfrutam de maior bem-estar subjetivo do que os que não o fazem ${ }^{(22)}$. A partir de certa idade muitas perdas acontecem: amigos se vão, os filhos constituem famílias, vão morar longe, entre outras. Suportar as mudanças requer boa auto-estima e apoio social. Dessa maneira, a velhice pode ser encarada como um momento de construir novas amizades e novas companhias.

Outro assunto geralmente de difícil discussão, principalmente para os idosos, é a morte, que traz consigo diferentes repercussões psicológicas associadas com a visão de transcendência. Em um estudo ${ }^{(23)}$ observaram que a maioria dos idosos entrevistados não considera a morte em si um tema muito preocupante, mas sim, o sofrimento que possa acompanhar o processo de morrer. Analisar a morte como um processo sofrido e/ou doloroso leva a pensar não só na pessoa que está chegando à sua finitude, mas também, nas pessoas que estão ao seu redor, como amigos, familiares, companheiros, que acabam sofrendo também com a situação de adoecimento e morte de seu ente querido. Pensar em adoecer, sofrer, sentir dor, causando assim tristeza nas pessoas que se ama, é também motivo que leva o idoso a pensar na morte como uma experiência ligada ao sofrimento ${ }^{(23-24)}$.

\section{CONSIDERAÇÕES FINAIS}

O presente estudo avaliou a qualidade de vida entre idosos em um bairro de Florianópolis, Santa Catarina, demonstrando que apesar de grande parte dos pesquisados apresentar um nível satisfatório de qualidade de vida, em alguns aspectos uma parcela considerável relatou insatisfação. Com a expectativa de vida da população cada vez maior, a atenção ao idoso deve ser também cada vez mais valorizada e qualificada. Além disso, acredita-se que a preocupação com o envelhecimento não deva ocorrer apenas na idade avançada, mas que seja tratada como um processo, para que se chegue à Terceira Idade com melhores condições de viver.

Buscar uma boa qualidade de vida para os idosos é também mudar a cultura sobre a visão da velhice, que ainda compreende o processo de envelhecimento como doença e fragilidade. Todavia, o fato de encontrar pessoas com mais de 60 anos em plena atividade laboral, realizando atividade física e com muitas responsabilidades, não é raro. Os idosos estão mais ativos e com uma esperança de vida maior, não apenas vivendo mais, mas também, com maior qualidade de vida. Com isso, muitos pré-conceitos em relação à velhice já não fazem parte da nossa realidade.

\section{AGRADECIMENTOS}

À Equipe de Saúde da Unidade Local de Saúde e aos idosos que participaram do estudo.

\section{REFERÊNCIAS}

1. Organización Mundial de la Salud. Grupo Científico sobre la epidemiología al estudio de los ancianos. Informe de un grupo científico de la OMS sobre la epidemiología Del envejecimiento. Ginebra: OMS; 1984.

2. Brasil. Lei n. 8.842, de 04 de Janeiro de 1994. Dispõe sobre a política nacional do idoso, cria o Conselho Nacional do Idoso e dá outras providências. Diário Oficial da República Federativa do Brasil, Brasília, 04 Jan. 1994.

3. IBGE. Perfil dos idosos responsáveis pelos domicílios no Brasil 2000. n.9. Rio de Janeiro, 2002. [acesso em 2007 Nov 04]. Disponível: http://www.ibge.gov.br/home/ estatistica/populacao/perfilidoso/perfidosos.2000.pdf

4. Camarano AA. Envelhecimento da população Brasileira: uma contribuição demográfica. Instituto de Pesquisa Econômica Aplicada 2002 jan. [acesso em 2009 Ago 06] Disponível: http://www.ipea.gov.br/pub/td/2002/ td_0858.pdf

5. Universidade Federal do Rio Grande do Sul. Avaliação da qualidade de vida. [acesso em 2008 Nov 04] Disponível: http://www.ufrgs.br/Psiq/whoqol.html. .

6. Jovtei EG. Qualidade de vida e o ensino tecnológico: uma experiência de pesquisa [dissertação]. Florianópolis (SC): Universidade Federal de Santa Catarina; 2001.

7. The World Health Quality of life Group. The World Health Organization quality of life assessment (WHOQOL): development and general psychometric properties. Soc Sci Med 1998; 46(12):1569-85.

8. Chachamovich E, Fleck MP, Trentini C, Power M. Brazilian WHOQOL-OLD module version: a rasch analysis of a new instrument. Rev Saúde Publ. 2008;42(2):308-16.

9. Fleck MPA, Chachamovich E, Trentini C. Development 
and validation of the Portuguese version of the WHOQOL-OLD module. Rev Saúde Publ. 2006;40(5):785-91.

10. Fleck MPA, Louzada S, Xavier M, Chachamovich E, Vieira G, Santos L, et al. Aplicação da versão em português do instrumento abreviado de avaliação da qualidade de vida "WHOQOL-bref” Rev Saúde Pública 2000; 34(2):178-83.

11. Neri AL. Qualidade de vida no adulto maduro: interpretações teóricas e evidências de pesquisa. In: Neri AL. Qualidade de vida e idade madura. Campinas: Papirus; 2006. p. 09-55.

12. LAROUSSE. Larousse da Terceira Idade. São Paulo/ SP: Larousse do Brasil, 2003.

13. Roach S. Introdução à Enfermagem Gerontológica. Rio de Janeiro: Guanabara Koogan; 2003.

14. Vitola J, Argimon IL. Aspectos psicológicos do envelhecimento. In: Terra NL, Dornelles B. Envelhecimento bem-sucedido. $2^{\mathrm{a}}$ ed. Porto Alegre: EdiPUCRS; 2003. p. 97-101.

15. Pires ZRS, Silva MJ. Autonomia e capacidade decisória dos idosos de baixa renda: uma problemática a ser considerada na saúde do idoso. Rev Eletron Enferm [periódico na Internet]. 2001 [acesso em 2009 Ago 06]3(2). Disponível: http://www.fen.ufg.br/revista/ revista3_2/autonomia.html

16. Bulla LC, Kaefer CO. Trabalho e aposentadoria: as repercussões sociais na vida do idoso aposentado. Texto Contexto Enferm. 2003; 2(1):34-46.

17. Agich GJ. Dependência e autonomia na velhice: um modelo ético para o cuidado de longo prazo. São Paulo: Edições Locais; 2008.

18. Portella MR. A utopia do envelhecer saudável nas ações coletivas dos grupos da terceira idade: canais de aprendizagem para a construção da cidadania. Texto Contexto Enferm. 2001; 10(2):196-202.

19. Paschoal SMP. Qualidade de vida na velhice. In: Freitas EV, PY L, Cançado FAX, Gorzoni ML, Rocha SM. Tratado de geriatria e gerontologia. Rio de Janeiro: Guanabara Koogan; 2002. p. 147-53.

20. Rolim FS, Forti VAM. Envelhecimento e atividade física: auxiliando na melhoria e manutenção da qualidade de vida. In: Cachioni M, Diogo MJD, Neri L, organizadores. Saúde e qualidade de vida na velhice. Campinas: Alínea; 2004, p. 57-73.
21. Lang FR, Staudinger UM, Carstensen LL. Perspectives on socioemotional selectivity in late life: how personality and social context (and do not) make a difference. J Gerontology Series B: Psychological Sciences and Social Sciences 1998; 53B(1):21-30.

22. Neri AL. Teorias psicológicas do envelhecimento. In: Freitas EV, Py L, Cançado FAX, Gorzoni ML, Rocha SM. Tratado de geriatria e gerontologia. Rio de Janeiro: Guanabara Koogan; 2002. p. 71-3.

23. Lunardi Filho WD, Sulzbach RC, Nunes AC, Lunardi VL. Percepções e condutas dos profissionais de enfermagem frente ao processo de morrer e morte. Texto Contexto Enferm. 2001;10(3):60-81.

24. Frumi C, Celich KLS. O olhar do idoso frente ao envelhecimento e à morte. Rev Bras Cienc Envelhec Humano. 2006; 3(2) 92-100. 